Pacific Journal of Mathematics

DERIVATIONS AND CAYLEY DERIVATIONS OF 


\section{DERIVATIONS AND CAYLEY DERIVATIONS OF GENERALIZED CAYLEY-DICKSON ALGEBRAS}

\section{KEVIN MCCRIMMON}

The Cayley-Dickson doubling process can be continued past the quaternions and octonions to obtain an infinite series of algebras of dimension $2^{n}$. After $n=3$ these algebras are no longer composition algebras. R. D. Schafer established the surprising result that the derivation algebras stop growing at $n=3$. Schafer's proof assumed the scalars were a field of characteristic $\neq 2,3$. In this paper we will give a different proof of his result which works for arbitrary rings of scalars, making use of the concept of a Cayley derivation.

Throughout, $A$ denotes a unital nonassociative algebra over an arbitrary (unital, commutative, associative) ring of scalars $\Phi$. We assume $A$ has a scalar involution * where all norms and traces are scalars,

$$
\begin{aligned}
& x x^{*}=N(x) 1, \quad x+x^{*}=T(x) 1, \\
& N(x, y)=T\left(x y^{*}\right), \quad T(x y)=T(y x) .
\end{aligned}
$$

If $\mu$ is a cancellable scalar ( $\mu a=0 \Rightarrow a=0$ ) then we can construct a new algebra with scalar involution by the Cayley-Dickson construction

$$
\begin{aligned}
& \mathbf{C}(A, \mu)=A \oplus A l, \\
& (a+b l)(c+d l)=\left(a c+\mu b^{*} d\right)+\left(d a+b c^{*}\right) l, \\
& (a+b l)^{*}=a^{*}-b l .
\end{aligned}
$$

The Cayley-Dickson process starts with $\Phi 1$ of dimension 1 and builds a *-extension $\Phi 1+\Phi w$ of dimension $2\left(w+w^{*}=1,1-4 N(w)\right.$ cancellable - if $\frac{1}{2} \notin \Phi$ we must take this by fiat for the second stage), then a quaternion algebra of dimension 4 , then an octonion algebra of dimension 8 ; the process continues to furnish algebras of dimension $2^{n}(n \geq 4)$, but these generalized Cayley-Dickson algebras are no longer alternative nor permit composition. Recall that the commuter $\operatorname{Comm}(A)$ consists of all elements commuting with $A$, the (left, middle, right) nuclei $N_{i}(A)$ consist of all elements associating with $A$, and the center $C(A)$ consists of all 
elements which commute and associate with $A$ :

$$
\begin{array}{ll}
\operatorname{Comm}(A)=\{c \mid[c, A]=0\}, & \\
N_{l}(A)=\{n \mid[n, A, A]=0\}, & N_{m}(A)=\{n \mid[A, n, A]=0\}, \\
N_{r}(A)=\{n \mid[A, A, n]=0\}, & N(A)=N_{l}(A) \cap N_{m}(A) \cap N_{r}(A), \\
C(A)=\operatorname{Comm}(A) \cap N(A), &
\end{array}
$$

where the commutator is $[x, y]=x y-y x$ and the associator is $[x, y, z]$ $=(x y) z-x(y z)$. For algebras with scalar involution we always have

$$
N_{l}(A)=N_{r}(A) \text {, }
$$

and for the generalized Cayley-Dickson algebras of dimension $2^{n}$ we have

$$
\begin{array}{ll}
N(x, y) \text { is nondegenerate } & \text { if } n \geq 1, \\
\operatorname{Comm}(A)=C(A)=\Phi 1 & \text { if } n \geq 2, \\
N(A)=\Phi 1 \quad \text { if } n \geq 3 &
\end{array}
$$

(see [4], 6.8-9).

Although the linearized norm form $N(x, y)=T\left(x y^{*}\right)$ is by $(0.7)$ usually nondegenerate, its radical will prove a nuisance later. The radical consists of the skew $*$-elements (cf. (1.1)),

$$
\operatorname{Rad} N(\cdot, \cdot)=\left\{z \mid z^{*}=-z, a z=z a^{*} \text { for all } a \in A\right\}
$$

since $N(z, 1)=0$ iff $z^{*}=-z$, and then $N(z, a)=0$ iff $z a^{*}-a z=0$. Any such nuclear $z$ 's kill commutators,

$$
z \in \operatorname{Rad} N(\cdot, \cdot) \cap N(A) \Rightarrow[A, A] z=0
$$

since

$$
[a, b] z=(a b) z-b(a z)=z\left(b^{*} a^{*}\right)-b\left(z a^{*}\right)=\left(z b^{*}-b z\right) a^{*}=0
$$

by nuclearity of $z$.

Any algebra with scalar involution has (generic) degree 2,

$$
x^{2}-T(x) x+N(x) 1=0,
$$

$\left(0.10^{\prime}\right) \quad x \circ y-T(x) y-T(y) x+N(x, y) 1=0 \quad(x \circ y=x y+y x)$.

A derivation of $A$ into a unital bimodule $M$ is a linear transformation $D$ : $A \rightarrow M$ such that

$$
D(x y)=D(x) y+x D(y) \text {. }
$$

The anti-derivations of $A$ into $M$ are just the derivations of $A$ into the opposite bimodule $M^{\mathrm{op}}$ (with $a \cdot_{\mathrm{op}} m=m a, m \cdot{ }_{\mathrm{op}} a=a m$ ), or from $A^{\mathrm{op}}$ into $M$,

$$
D(x y)=y D(x)+D(y) x .
$$


We denote by $\operatorname{Der}(A, M)$ and $\operatorname{Der}^{\mathrm{op}}(A, M)=\operatorname{Der}\left(A, M^{\mathrm{op}}\right)=$ $\operatorname{Der}\left(A^{\mathrm{op}}, M\right)$ the space of derivations and anti-derivations of $A$ into $M$. In the special case of the regular bimodule $M=A$, we denote the derivations and anti-derivations of $A$ by $\operatorname{Der}(A)$ and $\operatorname{Der}^{\mathrm{op}}(A)$.

Setting $x=y=1$ in $(0.11)$ or $\left(0.11^{\prime}\right)$ shows $D(1)=2 D(1)$, so

$$
D(1)=0, \quad D\left(x^{*}\right)=-D(x) \quad\left(D \in \operatorname{Der}^{\varepsilon}(A, M)\right) .
$$

If $D$ is a derivation or antiderivation of a degree 2 algebra into itself, setting $x=y$ in $(0.11)$ or $\left(0.11^{\prime}\right)$ shows

$$
\begin{aligned}
0 & =D\left(x^{2}\right)-D(x) \circ x \\
& =D(T(x) x)-T(x) D(x)-T(D(x)) x+N(D(x), x) 1 \\
& \left.\quad \text { (by }(0.10),\left(0.10^{\prime}\right),(0.12)\right) \\
& =-T(D(x)) x+N(D(x), x) 1,
\end{aligned}
$$

so derivations are traceless and skew

$$
\text { (0.13) } T(D x)=N(D x, x)=0 \quad\left(D \in \operatorname{Der}^{\varepsilon}(A), A\right. \text { rigid degree 2) }
$$

as long as $A$ is unitally faithful and rigid

$$
\alpha A=0 \Rightarrow \alpha=0 \text {, }
$$

(0.15) $F(x) x \in \Phi 1 \Rightarrow F=0$ if $F$ is a linear functional with $F(1)=0$.

(Note $F(x)=T(D x)$ has $F(1)=0$ by $(0.12)$ ). Assuming faithfulness (0.14) entails no loss of generality (pass to $\Phi / A^{\perp}$ ), and rigidity (0.15) holds in most reasonable cases (eg. if $\Phi$ has no nilpotent elements or $A$ is unitally free as $\Phi$-module ([4] 2.3)). From (0.3) we see $\mathbf{C}(A, \mu)$ is unitally faithful and rigid if $A$ is, in particular all generalized Cayley-Dickson algebras are faithful and rigid.

We will formulate our results quite generally for general (not-necessarily-rigid) algebras with scalar involution and algebras obtained from them by the Cayley-Dickson construction. The proofs would simplify considerably if we restricted ourselves to the case of generalized Cayley Dickson algebras.

1. Cayley derivations. If $A$ is an algebra with involution, a $*$-module is a bimodule $M$ consisting entirely of *-elements $m$

$$
a m=m a^{*} \quad \text { for all } a \in A \text {. }
$$

These are precisely the bimodules which become skew $*$-bimodules $((\mathrm{am}) *$ $\left.=m^{*} a^{*},(m a)^{*}=a^{*} m^{*}\right)$ under $m^{*}=-m$. There is a 1-1 correspondence between left, right, and *-modules for $A$. A Cayley derivation of $A$ into a 
*-module $M$ is a linear map $C$ such that

$$
C(x y)=C(x) y^{*}+C(y) x,
$$

and a Cayley anti-derivation is a Cayley derivation of $A$ into $M^{\text {op }}$

$$
C(x y)=C(x) y+C(y) x^{*} .
$$

We denote by $\operatorname{Cayder}(A)$ and $\operatorname{Cayder}^{\mathrm{op}}(A)$ the spaces of Cayley derivations and anti-derivations of $\boldsymbol{A}$ into itself (regarded as the regular right module). The archetypal example of a $*$-module is the Cayley-Dickson bimodule $\operatorname{Cay}(A)=A l$ as in (0.3); the importance of Cayley derivations is

$$
C \in \operatorname{Cayder}(A) \quad \text { iff } D \in \operatorname{Der}(A, \operatorname{Cay}(A)) \text { for } D(a)=C(a) l \text {. }
$$

Again setting $x=y=1$ in (1.2) or $\left(1.2^{\prime}\right)$ shows $C(1)=2 C(1)$,

$$
C(1)=0, \quad C\left(x^{*}\right)=-C(x) \quad\left(C \in \operatorname{Cayder}^{\varepsilon}(A)\right) .
$$

Unlike the derivation case (0.13), a Cayley derivation need not be traceless. We say $C$ is tracial if it has a trace element $c=t(C)$ such that

$$
T(C(x))=T(c x)=T(x c) .
$$

THE TRACE ELEMENT IS UNIQUELY DETERMINED ONLY IF $N(x, y)=T\left(x y^{*}\right)$ IS NONDEGENERATE; in general it is determined only up to an element of $\operatorname{Rad} N(\cdot, \cdot)$, which by $(0.8)$ means up to a skew $*$-element. For tracial $C$ any conjugate

$$
\hat{C}=C-R_{c} \quad(c=t(C), C \in t \text { Cayder }(A))
$$

has traceless range by $(1.5)$,

$$
T(\hat{C}(x))=0, \quad \hat{C}(x)^{*}=-\hat{C}(x)
$$

(by (1.4) $\hat{C}$ is never a Cayley derivation unless $t(C)=0$ ). Note that if $N(x, y)$ is nondegenerate over a field and $A$ is finite-dimensional, then all Cayley derivations are uniquely tracial: the linear functional $T(C(x))$ must be represented by a vector $c^{*}, T(C(x))=N\left(x, c^{*}\right)=T(x c)$. We denote by $t \operatorname{Cayder}(A)$ the space of tracial Cayley derivations of $A$, and by $n$ Cayder $(A)$ the space of Cayley derivations having a nuclear trace element $t(C) \in N(A)$.

\subsection{EXAMPLE. The standard skew Cayley map}

$$
S(x)=x^{*}-x
$$

is a (tracial) Cayley derivation of $A$ iff $3[A, A]=0$. Indeed,

$$
\begin{aligned}
S(x y)- & S(x) y^{*}-S(y) x \\
& =\left\{(x y)^{*}-x y\right\}-\left\{x^{*}-x\right\} y^{*}-\left\{y^{*}-y\right\} x \\
& =y^{*} x^{*}-x y-x^{*} y^{*}+x y^{*}-y^{*} x+y x \\
& =\left[y^{*}, x^{*}\right]-\left[y^{*}, x\right]+[y, x]=3[y, x]
\end{aligned}
$$


vanishes for all $x, y$ iff $3[A, A]=0$. We call it skew because its range is skew, so it has trace element $0: T(S(x))=T\left(x^{*}-x\right)=0$. If $A$ is commutative then $S$ is both a Cayley derivation and antiderivation, and if $A$ has characteristic 2 then $S$ is scalar-valued, $S(x)=x^{*}+x=T(x) 1 \in$ $\Phi 1$.

1.9. Proposition. If $A$ with scalar involution * either has (i) dimension $\geq 3$, or is unitally rigid with (ii) $\operatorname{Comm}(A)=\Phi 1$ or (iii) $A=\Phi 1+[A, A]$, then it admits no scalar-valued Cayley derivations or anti-derivations: $C(A)$ $\subset \Phi 1 \Rightarrow C=0$.

Proof. If $C(a)=F(a) 1$ for a linear functional $F$ satisfies (1.2) then $F(1)=0, F(a b) 1=F(a) b^{*}+F(b) a$. In case (i), if $1, a, b$ are independent we get $F(a)=F(b)=0$, so $F=C=0$. Applying $[a, \cdot]$ gives $0=$ [F(a)a, $b^{*}$ ], $F(a) a \in \operatorname{Comm}(A)$, so in case (ii) $F=C=0$ by unital rigidity (0.15). In case (iii) we apply $T(\cdot)$ to see $2 F(a b)=F(a) T(b)+$ $F(b) T(a)=F(T(b) a+T(a) b)=(a \circ b+N(a, b) 1)$ (by $\left.\left(0.10^{\prime}\right)\right)=$ $F(a b+b a)$, so $F([a, b])=0$ and $F$ vanishes on $[A, A]$ as well as $\Phi 1$, hence on $\Phi 1+[A, A]=A$ and again $F=C=0$. A similar argument applies to antiderivations.

1.10. Proposition. If $3 \Phi=0$ and $C$ is a Cayley derivation with $(C-\gamma)(A) \subset \Phi 1$ for some $\gamma$, then $C=\gamma S$ is a multiple of the standard skew Cayley map if either (i) $A$ has dimension $\geq 3$, or is unitally rigid with (ii) $\operatorname{Comm}(A)=\Phi 1$ or (iii) $A=\Phi 1+[A, A]$.

Proof. The condition (1.2) for $C(x)=\gamma x-F(x) 1$ ( $F$ a linear functional with $F(1)=\gamma$ ) becomes $\gamma x y-F(x y) 1=\gamma x y^{*}-F(x) y^{*}+\gamma y x-$ $F(y) x$, i.e. $0=\gamma\{T(y) x-x y\}-F(x)\{T(y) 1-y\}+\gamma\{x \circ y-x y\}$ $-F(y) x-\gamma x y+F(x y) 1=\{2 \gamma T(y)-F(y)\} x+\{\gamma T(y)+F(x)\} y+$ $\left\{-\gamma T\left(x y^{*}\right)+F(x y)-F(x) T(y)\right\} 1-3 \gamma x y$ (by $\left.\left(0.10^{\prime}\right)\right)$, so using $3 \gamma=0$ we see

$$
-H(y) x+H(x) y-H\left(x y^{*}\right) 1=0 \quad(H(x)=\gamma T(x)+F(x)) .
$$

Whenever $H=0$ we have $F(x)=-\gamma T(x), C(x)=\gamma x+\gamma\left(x+x^{*}\right)=$ $\gamma\left(x^{*}+2 x\right)=\gamma\left(x^{*}-x\right)=\gamma S(x)$, and $C=\gamma S$. (i) If $\operatorname{dim} A \geq 3$ we take $x, y, 1$ independent in (1.11) to see $H=0$. (ii) We commute (1.11) with $x$ to get $H(x)[x, y]=0, H(x) x \in \operatorname{Comm}(A)=\Phi 1$ with $H(1)=3 \gamma=0$, so if $A$ is unitally rigid as in (0.15) we see $H=0$. (iii) Since $\frac{1}{2}=-1$ in 
characteristic 3 we can write $A=\Phi 1 \oplus A_{0}\left(T\left(A_{0}\right)=0\right)$, so $-H\left(y_{0}\right) x_{0}+$ $H\left(x_{0}\right) y_{0}=-H\left(x_{0} y_{0}\right) 1$ in (1.11) implies $H\left(x_{0} y_{0}\right)=0, H\left(x_{0}\right) y_{0}=$ $H\left(y_{0}\right) x_{0}$ for $x_{0}, y_{0}$ in $A_{0}$, in particular

$$
H(A)[A, A]=H\left(A_{0}\right)\left[A_{0}, A_{0}\right]=H\left(\left[A_{0}, A_{0}\right]\right) A_{0}=0
$$

since $[A, A] \subset A_{0}$ by $(0.2)$, so $H([A, A])=H\left(\left[A_{0}, A_{0}\right]\right)=0$ and already $H(1)=1$, therefore $H(A)=0$ and $H=0$.

1.12 EXAMPLE. If $A$ is associative with scalar involution, then

$$
C(x)=\sum a_{i} x b_{i}+T(x) d
$$

is a Cayley derivation if $d=\sum a_{i} b_{t}^{*}$ and $0=\sum a_{i}\left(b_{i}+2 b_{t}^{*}\right)$, in which case $C$ has trace element $t(C)=\sum b_{i} a_{i}+T(d) 1$. As a special case, if $(a b)^{*}=$ $a b^{*}$ (eg. if $b$ and $a b$ are skew) then

$$
C(x)=[a, x] b \quad\left((a b)^{*}=a b^{*}\right)
$$

is a Cayley derivation with trace element $t(C)=[b, a]$ Indeed, if $\Delta F(x, y)=F(x y)-F(x) y^{*}-F(y) x$ measures how far $F$ is from being a Cayley derivation, then for $E_{a, b}(x)=a x b$ and $T_{d}(x)=T(x) d$ we have

$$
\Delta E_{a, b}(x, y)=a b^{*}[x, y]-a b x y^{*}, \quad \Delta T_{d}(x, y)=-d[x, y]-2 d x y^{*},
$$

so $C=\sum E_{a_{t}, b_{t}}+T_{d}$ has

$$
\Delta C(x, y)=\left\{\sum a_{i} b_{i}^{*}-d\right\}[x, y]-\left\{\sum a_{i} b_{i}+2 d\right\} x y^{*} .
$$

2. Derivations of $\mathbf{C}(A, \mu)$. In this section we show how the derivations of $\mathbf{C}(A, \mu)$ are built out of derivations, Cayley derivations, and skew nuclear elements of $A$. An immediate calculation from the definition (0.11) of derivation and the definition (0.4) of the product on $\mathbf{C}$ shows that every $*$-derivation of $A\left(D\left(a^{*}\right)=D(a)^{*}\right)$ extends to one of $\mathbf{C}$. The *-condition is just that $D(a)^{*}=-D(a)$, i.e. that $D$ be traceless $T(D(a))$ $=0$. We noted in $(0.13)$ that all derivations are traceless in the unitally rigid case. Our calculations could be simplified if we assumed unital rigidity.

2.1 Lemma. $A$ map $D_{+}(a+b l)=D_{11}(a)+D_{22}(b) l$ is a derivation of $\mathrm{C}(A, \mu)$ iff

(i) $D_{11}=D_{0}$ is a traceless derivation of $A$

(ii) $D_{22}=D_{0}+L_{d_{0}}$ for a skew element $d_{0}$ in the nucleus of $A$.

Proof. $D_{+}$restricts to a derivation of $A$ into $A+A l$, hence its projection $D_{11}$ into the submodule of $A$ must be a derivation $D_{0}$ of $A$. 
Then the cierivation condition $D\left(x_{1} x_{2}\right)=D\left(x_{1}\right) x_{2}+x_{1} D\left(x_{2}\right)$ for $x_{i}=$ $a_{i}+b_{i} l$ reduces to

$$
\begin{aligned}
D_{0}\left(a_{1} a_{2}+\mu b_{2}^{*} b_{1}\right)+D_{22}\left(b_{1} a_{2}^{*}+b_{2} a_{1}\right) l \\
=\left\{D_{0}\left(a_{1}\right) a_{2}+a_{1} D_{0}\left(a_{2}\right)\right\}+\mu\left\{b_{2}^{*} D_{22}\left(b_{1}\right)+D_{22}\left(b_{2}\right)^{*} b_{1}\right\} \\
\quad+\left\{b_{2} D_{0}\left(a_{1}\right)+D_{22}\left(b_{2}\right) a_{1}\right\} l+\left\{D_{22}\left(b_{1}\right) a_{2}^{*}+b_{1} D_{0}\left(a_{2}\right)^{*}\right\} l,
\end{aligned}
$$

i.e.

$$
\begin{aligned}
& D_{22}\left(b_{1} a_{2}^{*}\right)=D_{22}\left(b_{1}\right) a_{2}^{*}+b_{1} D_{0}\left(a_{2}\right)^{*} \\
& D_{22}\left(b_{2} a_{1}\right)=D_{22}\left(b_{2}\right) a_{1}+b_{2} D_{0}\left(a_{1}\right) \\
& D_{0}\left(b_{2}^{*} b_{1}\right)=b_{2}^{*} D_{22}\left(b_{1}\right)+D_{22}\left(b_{2}\right)^{*} b_{1} .
\end{aligned}
$$

Setting $d_{0}=D_{22}(1)$, we see by (2) that $D_{22}(a)=d_{0} a+D_{0}(a)$, so $D_{22}=$ $D_{0}+L_{d_{0}}$ as in (ii), and (2) reduces to left nuclearity $d_{0}(b a)=\left(d_{0} b\right) a$ of $d_{0}$. (1) $+(2)$ reduces to $D_{22}(b T(a))=D_{22}(b) T(a)+b T\left(D_{0}(a)\right)$, which is just tracelessness of $D_{0}$ as in (i). Hence (3) reduces to $0=b_{2}^{*}\left(d_{0} b_{1}\right)+$ $\left(b_{2}^{*} d_{0}^{*}\right) b_{1}$, which for $b_{1}=b_{2}=1$ yields skewness $d_{0}+d_{0}^{*}=0$, and therefore (3) is middle nuclearity $0=-\left[b_{2}^{*}, d_{0}, b_{1}\right]$ of $d_{0}$. iff

2.2. LemMA. $D_{-}(a+b l)=D_{12}(b)+D_{21}(a) l$ is a derivation of $C(A, \mu)$

(i) $D_{21}=C_{0}$ is a Cayley derivation of $A$ such that $\mu C_{0}$ has a skew nuclear trace element $c_{0}$

(ii) $D_{12}=\frac{\mu C_{0}}{\mu C_{0}}-R_{c_{0}}$.

Proof. If $D_{-}$is a derivation of $C$ then its restriction to $A$ and projection into $A l$ gives a derivation of $A$ into $A l$, so by (1.3) $D_{21}$ is a Cayley derivation $C_{0}$. The derivation condition (0.11) for $x_{i}=a_{i}+b_{i} l$ becomes

$$
\begin{aligned}
D_{12}\left(b_{1} a_{2}^{*}+b_{2} a_{1}\right)+C_{0}\left(a_{1} a_{2}+\mu b_{2}^{*} b_{1}\right) l \\
=\left\{D_{12}\left(b_{1}\right) a_{2}+\mu b_{2}^{*} C_{0}\left(a_{1}\right)\right\}+\left\{C_{0}\left(a_{1}\right) a_{2}^{*}+b_{2} D_{12}\left(b_{1}\right)\right\} l \\
\quad+\left\{a_{1} D_{12}\left(b_{2}\right)+\mu C_{0}\left(a_{2}\right)^{*} b_{1}\right\}+\left\{C_{0}\left(a_{2}\right) a_{1}+b_{1} D_{12}\left(b_{2}\right)^{*}\right\} l,
\end{aligned}
$$

i.e.,

$$
\begin{aligned}
& D_{12}\left(b_{2} a_{1}\right)=\mu b_{2}^{*} C_{0}\left(a_{1}\right)+a_{1} D_{12}\left(b_{2}\right) \\
& D_{12}\left(b_{1} a_{2}^{*}\right)=D_{12}\left(b_{1}\right) a_{2}+\mu C_{0}\left(a_{2}\right)^{*} b_{1} \\
& \mu C_{0}\left(b_{2}^{*} b_{1}\right)=b_{2} D_{12}\left(b_{1}\right)+b_{1} D_{12}\left(b_{2}\right)^{*}
\end{aligned}
$$


If we let $c_{0}=-D_{12}(1), C=\mu C_{0}$ then $b_{2}=1$ in (1) yields $D_{12}(a)=C(a)$ - $a c_{0}$, so $D_{12}=C-R_{c_{0}}$ as in (ii). Setting $a_{2}=b_{2}=1$ in (3) yields $c_{0}+c_{0}^{*}=0$ (using (1.4)), so $b_{1}=1$ in (2) yields

$$
\begin{aligned}
0 & =C\left(a^{*}\right)-a^{*} c_{0}+c_{0} a-C(a)^{*} \\
& =-C(a)+a^{*} c_{0}^{*}+c_{0} a-C(a)^{*} \quad(\text { by }(1.4)) \\
& =T\left(c_{0} a-C(a)\right),
\end{aligned}
$$

so $C$ is tracial with trace element $c_{0}$ as in (1.5) and conjugate $\hat{C}=D_{12}$ as in (1.6). Thus (2) becomes

$$
\begin{aligned}
0=C\left(b a^{*}\right)+\left(b a^{*}\right) c_{0}^{*}-C(b) a+\left(b c_{0}\right) a-T\left(c_{0} a\right) b-C\left(a^{*}\right) b \\
\left.\quad \quad \text { using skewness of } c_{0},(1.5),(1.4)\right) \\
=\left[b, a^{*}, c_{0}^{*}\right]+b\left(a^{*} c_{0}^{*}\right)+\left[b, c_{0}, a\right]+b\left(c_{0} a\right)-b T\left(c_{0} a\right) \\
=\left[b, a, c_{0}\right]+\left[b, c_{0}, a\right],
\end{aligned}
$$

(1) $+(2)$ becomes

$$
\begin{aligned}
0 & =T(a) \hat{C}(b)-a \circ \hat{C}(b)-N(C(a), b) 1 \\
& =N(a, \hat{C}(b))-N(C(a), b) \quad\left(\text { by }\left(0.10^{\prime}\right),(1.7)\right) \\
& =-T\left(\left[a, b, c_{0}\right]\right)
\end{aligned}
$$

(see (2.10) below), (1) + (3) becomes

$$
\begin{aligned}
0 & =\left\{\hat{C}(b a)-b^{*} C(a)-a \hat{C}(b)\right\}+\left\{C\left(b a^{*}\right)-b \hat{C}(a)-a \hat{C}(b)^{*}\right\} \\
& =C(T(b) a)-(b a) c_{0}-T(b) C(a)+b\left(a c_{0}\right) \quad(\text { by }(1.7)) \\
& =-\left[b, a, c_{0}\right] .
\end{aligned}
$$

Therefore (1)-(3) hold iff $D_{12}=\hat{C}$ where the trace $c_{0}$ of $C$ is right $=$ left nuclear and middle nuclear, i.e. $c_{0}$ is skew nuclear.

2.3. Corollary. $D(a+b l)=b z_{0}$ is a derivation of $\mathbf{C}(A, \mu)$ iff $z_{0} \in$ $\operatorname{Rad} N(\cdot, \cdot) \cap N(A)$ is radical in the nucleus, i.e. a skew nuclear *-element.

Proof. This is the case $C_{0}=C=0$ of the Lemma, $c_{0}=z_{0}$ is any skew nuclear trace element for $0: T\left(z_{0} x\right)=0$ for all $x$, i.e. (by $\left.(0.2)\right) N\left(z_{0}, A\right)$ $=0$ and we apply the characterization (0.8).

2.4. Derivation Theorem. If $A$ is an algebra with scalar involution and $\mu$ a cancellable scalar, then the derivations of $\mathbf{C}(A, \mu)=A \oplus$ Al are precisely all

$$
D=\left(\begin{array}{ll}
D_{0} & C_{0}-R_{c_{0}}-R_{z_{0}} \\
C_{0} & D_{0}+L_{d_{0}}
\end{array}\right)=\tilde{D}_{0}+\tilde{C}_{0}+\tilde{d}_{0}+\tilde{z}_{0}
$$


where

(i) $D_{0}$ is a traceless derivation of $A$

(ii) $d_{0} \in N_{0}(A)$ is skew nuclear

(iii) $C_{0}$ is a Cayley derivation of $A$ such that $\mu C_{0}$ has skew nuclear trace element $c_{0} \in N_{0}(A)$

(iv) $z_{0} \in N_{\mathrm{rad}}(A)$ is radical in the nucleus (skew nuclear *-element).

We have the Schafer decomposition

$$
\operatorname{Der}(\mathbf{C}(A, \mu))=\mathscr{D}_{0} \oplus \mathscr{N}_{0} \oplus \mathscr{C}_{0} \oplus \mathscr{Z}_{0}
$$

where

$$
D_{0} \rightarrow \tilde{D}_{0}=\left(\begin{array}{ll}
D_{0} & 0 \\
0 & D_{0}
\end{array}\right)
$$

is a bijection of $\operatorname{Der}_{0}(A)$ onto $\mathscr{D}_{0}$,

$$
d_{0} \rightarrow \tilde{d}_{0}=\left(\begin{array}{ll}
0 & 0 \\
0 & L_{d_{0}}
\end{array}\right)
$$

is a bijection of $N_{0}(A)$ onto $\mathscr{N}_{0}$,

$$
C_{0}+z_{0} \rightarrow \tilde{C}_{0}+\tilde{z}_{0}=\left(\begin{array}{ll}
0 & C_{0}-R_{c_{0}}-R_{z_{0}} \\
C_{0} & 0
\end{array}\right)
$$

is a bijection of $\operatorname{Cayder}_{\mu}(A)+N_{\text {rad }}(A)$ onto $\mathscr{C}_{0} \oplus \mathscr{Z}_{0}$, with multiplication rules

$$
\begin{aligned}
& {\left[\tilde{D}_{0}, \tilde{D}_{0}^{\prime}\right]=\left[\widetilde{D_{0}, D_{0}^{\prime}}\right] \in \mathscr{D}_{0}, \quad\left[\tilde{d}_{0}, \tilde{d}_{0}^{\prime}\right]=\left[\overline{d_{0}, d_{0}^{\prime}}\right] \in \mathscr{N}_{0},} \\
& {\left[\tilde{D}_{0}, \tilde{C}_{0}\right]=\left[\widetilde{D_{0}, C_{0}}\right] \in \mathscr{C}_{0}, \quad\left[\tilde{d}_{0}, \tilde{C}_{0}\right]=\widetilde{L_{d_{0}} C_{0}} \in \mathscr{C}_{0},} \\
& \text { (2.7) }\left[\tilde{D}_{0}, \tilde{d}_{0}\right]=\widetilde{D_{0}\left(d_{0}\right)} \in \mathscr{N}_{0}, \quad\left[\tilde{d}_{0}, \tilde{z}_{0}\right]=-\widetilde{d_{0} z_{0}} \in \mathscr{Z}_{0} \text {, } \\
& {\left[\tilde{C}_{0}, \tilde{C}_{0}^{\prime}\right]=\tilde{D}_{0}+\tilde{d}_{0} \quad\left(D_{0}=\widehat{\mu C_{0}^{\prime}} C_{0}^{\prime}-\widehat{\mu C_{0}^{\prime}} C_{0} \in \operatorname{Der}(A),\right.} \\
& \left.d_{0}=C_{0}^{\prime}\left(c_{0}\right)-C_{0}\left(c_{0}^{\prime}\right) \in N_{0}(A)\right) \\
& {\left[\tilde{C}_{0}, \tilde{z}_{0}\right]=\tilde{D}_{0}+\tilde{d}_{0} \quad\left(D_{0}=-R_{z_{0}} C_{0} \in \operatorname{Der}(A), d_{0}=C_{0}\left(z_{0}\right) \in N_{0}(A)\right) .}
\end{aligned}
$$

Proof. $\mathbf{C}(A, \mu)=A \oplus A l$ is a $\mathbf{Z}_{2}$-graded algebra, and

$$
D=\left(\begin{array}{ll}
D_{11} & D_{12} \\
D_{21} & D_{22}
\end{array}\right)
$$


is a derivation iff its even and odd parts

$$
D_{+}=\left(\begin{array}{ll}
D_{11} & 0 \\
0 & D_{22}
\end{array}\right) \quad\left(D_{+}(a+b l)=D_{11}(a)+D_{22}(b) l\right)
$$

and

$$
D_{-}=\left(\begin{array}{ll}
0 & D_{12} \\
D_{21} & 0
\end{array}\right) \quad\left(D_{-}(a+b l)=D_{12}(b)+D_{21}(a) l\right)
$$

are derivations. Here $D_{+}$is a derivation iff $D_{+}=\tilde{D}_{0}+\tilde{d}_{0}$ as in (i), (ii) by Lemma 2.1, and $D_{-}$is a derivation iff $D_{-}=\tilde{C}_{0}+\tilde{z}_{0}$ as in (iii), (iv) by Lemma 2.2 (any two nuclear trace elements for $\mu C_{0}$ differ by a nuclear radical element $z_{0}$ ) and Corollary 2.3. The multiplication rules (2.7) follow from direct matrix calculation, noting that if

$$
D=\left(\begin{array}{ll}
D_{11} & D_{12} \\
D_{21} & D_{22}
\end{array}\right)
$$

then $d_{0}=D_{22}(1), c_{0}+z_{0}=D_{12}(1), D_{0}=D_{11}, C_{0}=D_{21}$ in (2.5) (so, for example ,

$$
\left[\tilde{D}_{0}, \tilde{z}_{0}\right]=\left(\begin{array}{ll}
0 & {\left[D_{0}, R_{z_{0}}\right]} \\
0 & 0
\end{array}\right)=\left(\begin{array}{ll}
0 & R_{D_{0}\left(z_{0}\right)} \\
0 & 0
\end{array}\right)
$$

must have the form

$$
\left(\begin{array}{ll}
0 & R_{w_{0}} \\
0 & 0
\end{array}\right) \quad \text { in } \mathscr{Z}_{0}
$$

so $\left.w_{0}=D_{0}\left(z_{0}\right) \in N_{\text {rad }}(A)\right)$.

We call 2.6 the Schafer decomposition of $\operatorname{Der}(\mathbf{C}(A, \mu))$ since it was first noticed by R. D. Schafer [5, p. 66] for the case when $A$ is a quaternion algebra and $\mathbf{C}(A, \mu)$ a Cayley algebra, and was used to analyze the Lie algebra $\operatorname{Der}(\mathbf{C})$ of type $G_{2}$.

When $A$ has no Cayley derivations, $\mathbf{C}(A, \mu)$ has essentially the same derivations as $A$.

2.8. Corollary. If $A$ has $N(A)=\Phi 1$ and $N(x, y)$ nondegenerate, then

(i) when $A$ has no 2 or 3-torsion and Cayder $(A)=0$ we have

$$
\operatorname{Der}(\mathbf{C}(A, \mu))=\widetilde{\operatorname{Der}_{0}(A)}
$$


(ii) when $2 A=0$ and Cayder $(A)=0$ we have

$\operatorname{Der}(\mathbf{C}(A, \mu))=\widehat{\operatorname{Der}_{0}(A)} \boxplus \Phi Z \quad(Z(a+b l)=b l$ central $)$

(iii) when $3 A=0$ and Cayder $(A)=\Phi S$ we have

$$
\begin{gathered}
\operatorname{Der}(\mathbf{C}(A, \mu))=\widetilde{\operatorname{Der}_{0}(A)} \boxplus \Phi W \\
\left(W(a+b l)=\mu S(b)+S(a) l \text { central }, S(a)=a^{*}-a\right) .
\end{gathered}
$$

Proof. We know any $D \in \operatorname{Der}(\mathbf{C}(A, \mu))$ has the form $\tilde{D}_{0}+\tilde{d}_{0}+\tilde{C}_{0}$ $+\tilde{z}_{0}$; if $N(x, y)$ is nondegenerate then $N_{\text {rad }}(A)=0, \tilde{z}_{0}=0$; if $N(A)=\Phi 1$ then $d_{0} \in \Phi 1$ is skew iff $2 d_{0}=0$, so $d_{0}=0$ when $A$ has no 2-torsion and $d_{0}=\delta 1$ is arbitrary if $2 A=0$, with $\left[\tilde{D}_{0}, \tilde{d}_{0}\right]=\widetilde{D_{0}\left(d_{0}\right)}=\widehat{D_{0}(1)}=0$ by (2.7), (0.12). By hypothesis $C_{0}=0$ in the first two cases and $C_{0}=\gamma S$ in case (iii), with $\left[\tilde{D}_{0}, \tilde{S}\right]=\left[\widetilde{D_{0}, S}\right]=0$ by $(2.7)$ and $\left[D_{0}, S\right](a)=$ $D_{0}\left(a^{*}-a\right)-\left\{D_{0}(a)^{*}-D_{0}(a)\right\}=0$ if $D_{0}$ is traceless.

2.9 REMARK. As a consequence of the multiplication rules (2.7) we immediately obtain

(i) If $D_{0} \in \operatorname{Der}_{0}(A), C_{0} \in n \operatorname{Cayder}(A)$ then $\left[D_{0}, C_{0}\right] \in n$ Cayder $(A)$ with $\left[\widehat{D_{0}, C_{0}}\right]=\left[D_{0}, \hat{C}_{0}\right], t\left(\left[D_{0}, C_{0}\right]\right)=D_{0}\left(t\left(C_{0}\right)\right)$;

(ii) If $D_{0} \in \operatorname{Der}_{0}(A), d_{0} \in N_{0}(A), z_{0} \in N_{\text {rad }}(A)$ then $D_{0}\left(d_{0}\right) \in$ $N_{0}(A), D_{0}\left(z_{0}\right) \in N_{\text {rad }}(A)$;

(iii) If $d \in N_{0}(A), C \in n \operatorname{Cayder}(A)$ then $L_{d} C \in n \operatorname{Cayder}(A)$ with $\widehat{L_{d} C}=-\hat{C} L_{d}, t\left(L_{d} C\right)=\hat{C}(d)=C(d)-d t(C) \in N_{0}(A)$

(iv) If $d \in N_{0}(A), z \in N_{\mathrm{rad}}(A)$ then $R_{z} L_{d}=R_{d z}$ for $d z \in N_{\mathrm{rad}}(A)$;

(v) If $C, C^{\prime} \in n$ Cayder $(A)$ then $D=\hat{C} C^{\prime}-\hat{C}^{\prime} C \in \operatorname{Der}(A)$ and $C \hat{C}^{\prime}-C^{\prime} \hat{C}=D+L_{d}$ for $d=C^{\prime}(t(C))-C\left(t\left(C^{\prime}\right)\right) \in N_{0}(A)$;

(vi) If $C \in n$ Cayder $(A), z \in N_{\text {rad }}(A)$ then $D=R_{z} C \in \operatorname{Der}(A)$ with $R_{z} C+C R_{z}=L_{d}$ for $d=C(z) \in N_{0}(A)$.

These of course can all be proven directly from the definitions, though at the expense of considerable effort. Direct calculation often yields slightly stronger statements, as we will now indicate. For computing trace elements it will be convenient to note that $\hat{C}$ is the adjoint of $C$ when $t(C)$ is nuclear; more generally

(2.10) $N(C x, y)-N(x, \hat{C}(y))=T([x, y, t(C)]) \quad(C \in t$ Cayder $(A))$ since

$$
\begin{aligned}
& T\left(C(x) y^{*}\right)-T\left(C(y) x^{*}\right)+T\left(x^{*}(y c)\right) \quad(\text { using }(0.2), c=t(C)) \\
& \quad=T\left(\left(x^{*} y\right) c-\left[x^{*}, y, c\right]-C\left(x^{*} y\right)\right) \quad(\text { by }(1.4)) \\
& \quad=T([x, y, c]) \quad(\text { by }(1.5)) .
\end{aligned}
$$


Improving on (i), from the definitions (0.11) and (1.2) we see

(i') If $D_{0} \in \operatorname{Der}_{0}(A), C \in \operatorname{Cayder}(A)$ then $\left[D_{0}, C\right] \in \operatorname{Cayder}(A)$; if $C$ is tracial so is $\left[D_{0}, C\right]$ with $\left[\widehat{D_{0}, C}\right]=\left[D_{0}, \hat{C}\right]$ and $t\left(\left[D_{0}, C\right]\right)=$ $D_{0}(t(C))$

(we need $D_{0}$ traceless for it to be a $*$-derivation); note that if $c=t(C)$ then $T([D, C] x)=-T(C(D x))=-T(c D(x))=T(D(c) x-D(c x))=$ $T(D(c) x)$ when $D$ is traceless. Here $D(c)$ is nuclear if $c$ is since derivations preserve nuclei, is skew if $D$ is traceless, and is a $*$-element if $c$ is (as in (ii)). For the conjugate (1.6), note $\left[D, R_{c}\right]=R_{D(c)}$ for any derivatives by $(0.11)$.

Improving upon (iii), we have directly from (1.2) that

(iii') If $d \in N_{l}(A), C \in \operatorname{Cayder}(A)$ then $L_{d} C \in \operatorname{Cayder}(A)$; if $d$ is nuclear and $C$ is tracial so is $L_{d} C$, with $\widehat{L_{d} C}=\hat{C} L_{d^{*}}, t\left(L_{d} C\right)=$ $-\hat{C}\left(d^{*}\right)$.

For the trace, when $d$ is nuclear $T(d C(x))=N\left(d^{*}, C(x)\right)=N\left(\hat{C}\left(d^{*}\right), x\right)$ (by $(2.10))=T\left(\hat{C}\left(d^{*}\right)^{*} x\right) \quad($ by $(0.2))=-T\left(\hat{C}\left(d^{*}\right) x\right) \quad$ (by (1.7)) with $-\hat{C}\left(d^{*}\right)=-C\left(d^{*}\right)+d^{*} c=C(d)+d^{*} c$ by (1.4). For the conjugate,

$\left\{\hat{C} L_{d^{*}}-\widehat{L_{d} C}\right\}(x)=\hat{C}\left(d^{*} x\right)+\widehat{L_{d} C}(x)^{*} \quad($ by $(1.7))$

$=C\left(d^{*} x\right)+\left\{d C(x)-x\left(c d-C(d)^{*}\right)\right\}^{*}$

$=C\left(d^{*} x\right)-d^{*} x c+C(x)^{*} d^{*}-d^{*} c^{*} x^{*}+C(d) x^{*} \quad\left(d^{*}\right.$ is left nuclear $)$

$=T(C(x)) d^{*}-d^{*}\left(x c+c^{*} x^{*}\right) \quad($ by $(1.2),(1.4))$

$=0 \quad($ by $(1.5))$.

If $c$ is nuclear so is $\hat{C}(d)$, i.e. $C(d)$ is, by the nontrivial calculation in Lemma 2.11 below.

Improving on (iv), we have

(iv') If $z$ is a skew *-element then $R_{z} L_{d}=L_{d} R_{z}=R_{d z}$ for $d z$ a skew *-element when either $d$ or $z$ is nuclear; if both are nuclear, so is $d z$.

Indeed, by (0.9) $d x z=x d z$ where $T(d z)=0, a d z=z d^{*} a^{*}=d z a^{*}$ if one of $d, z$ is nuclear.

To see why the first part of (v) should hold, if $c_{i}=t\left(C_{i}\right)$ are nuclear then

$$
\begin{aligned}
& \hat{C}_{1} C_{2}(x y)-\hat{C}_{1} C_{2}(x) y-x \hat{C}_{1} C_{2}(y) \\
& \quad=\hat{C}_{1}\left\{C_{2}(x) y^{*}+C_{2}(y) x\right\}-\hat{C}_{1} C_{2}(x) y+x\left\{\hat{C}_{1} C_{2}(y)\right\}^{*}
\end{aligned}
$$




$$
\begin{aligned}
= & \left\{C_{1} C_{2}(x) y-C_{1}(y) C_{2}(x)-C_{2}(x) y^{*} c_{1}\right\} \\
& +\left\{C_{1} C_{2}(y) x^{*}+C_{1}(x) C_{2}(y)-C_{2}(y) x c_{1}\right\} \\
& -\hat{C}_{1} C_{2}(x) y-\hat{C}_{1} C_{2}(y) x^{*}+N\left(x, \hat{C}_{1} C_{2}(y)\right) \quad(\text { by }(1.2),(1.4)) \\
= & C_{1}(x) C_{2}(y)-C_{1}(y) C_{2}(x)+C_{2}(x)\left(c_{1} y+y^{*} c_{1}^{*}\right) \\
& -C_{2}(y)\left(x c_{1}+c_{1}^{*} x^{*}\right)+N\left(C_{2}(x), C_{2}(y)\right) \\
& \quad\left(\text { by }(1.6), \text { skewness of } c_{1}, \text { and }(2.10)\right) \\
= & \left\{T\left(c_{1} y\right)-C_{1} y\right\} C_{2}(x)-\left\{T\left(x c_{1}\right)-C_{1}(x)\right\} C_{2}(y) \\
& +C_{1}(x)^{*} C_{2}(y)+C_{2}(y)^{*} C_{1}(x) \\
= & C_{1}(y)^{*} C_{2}(x)+C_{2}(y)^{*} C_{1}(x)
\end{aligned}
$$

(by (1.6), skewness of $c_{1}$, and (2.10))

is symmetric in the indices 1 and 2 , therefore $D=\hat{C}_{1} C_{2}-\hat{C}_{2} C_{1}$ has $D(x y)-D(x) y-x D(y)=0$ and $D$ is a derivation. For the second part of $(\mathrm{v})$, note that

$$
\begin{aligned}
& \left(C_{1} \hat{C}_{2}-C_{2} \hat{C}_{1}\right)-\left(\hat{C}_{1} C_{2}-\hat{C}_{2} C_{1}\right) \\
& \quad=\left(-C_{1} R_{c_{2}}+C_{2} R_{c_{1}}\right)-\left(-R_{c_{1}} C_{2}+R_{c_{2}} C_{1}\right) \\
& \quad=\left(C_{2} R_{c_{1}}+R_{c_{1}} C_{2}\right)-\left(C_{1} R_{c_{2}}+R_{c_{2}} C_{1}\right)=L_{C_{2}\left(c_{1}\right)}-L_{C_{1}\left(c_{2}\right)}
\end{aligned}
$$

for skew $c_{i}$ by (1.2).

Improving on (vi), a direct calculation using (0.8), (0.9) and nuclearity of $z$ shows

(vi') If $C$ is a Cayley derivation and $z$ is a skew nuclear $*$-element, then $D=R_{z} C$ is a derivation with $C R_{z}=L_{C(z)}-D$; if $C$ is tracial then $C(z)$ is skew nuclear.

Note by (1.2) we have $R_{z} C+C R_{z}=L_{C(z)}$ whenever $z$ is skew. Certainly $C(z)$ is skew if $C$ is tracial, $T(C(z))=T(c z)=0$ for radical $z$, and $C(z)$ is nuclear by the following Lemma 2.11 .

It seems to be difficult to prove directly that Cayley derivations preserve the nucleus.

2.11. Lemma. If $d \in N(A)$ is nuclear and $C$ a Cayley derivation, then $C(d) \in N_{l}(A)=N_{r}(A)$ is outer-nuclear. If $C$ has a nuclear trace element then $C(d) \in N(A)$ is also middle-nuclear.

Proof. For any Cayley derivation we have

$$
C([x, y, y])=[C(x), y, y]-[C(y), x, y]
$$


since

$$
\begin{aligned}
& C\left(-\left[x, y, y^{*}\right]\right)+\left[C(x), y^{*}, y\right]+[C(y), x, y] \\
&=-C\left((x y) y^{*}\right)+C(x N(y))+\left\{C(x) y^{*}\right\} y-C(x) N(y) \\
&+\{C(y) x\} y+C\left(y^{*}\right)(x y) \quad(\text { by }(1.4)) \\
&=\left\{-C(x y)+C(x) y^{*}+C(y) x\right\} y=0 \quad \text { (by (1.2). }
\end{aligned}
$$

Linearizing $y \rightarrow y, d$ for nuclear $d$ shows $0=-[C(d), x, y]$, i.e. $C(d) \in$ $N_{l}(A)$.

When $C$ has nuclear trace element $c$ we have

$$
\begin{aligned}
& \hat{C}(x y)=\hat{C}(x) y^{*}-C(y) * x \\
& \hat{C}(x y)=x^{*} C(y)+y \hat{C}(x)
\end{aligned}
$$

since direct calculation from (1.2), (1.6), (1.7) shows that

$$
\begin{aligned}
\hat{C}(x y)-\hat{C}(x) y^{*}+C(y)^{*} x & =-x y c+x c y^{*}+T(C(y)) x \\
& =x\left\{T(y c)-y c-c^{*} y^{*}\right\}=0
\end{aligned}
$$

and $\left\{\hat{C}(x) y^{*}-C(y)^{*} x\right\}-\left\{x^{*} C(y)+y \hat{C}(x)\right\}=T(y) \hat{C}(x)-y \circ \hat{C}(x)$ $-N(C(y), x)=0$ by $\left(0.10^{\prime}\right),(1.7),(2.10)$. Then

$$
\begin{aligned}
& {\left[x^{*}, C(d), y\right]=\left\{x^{*} C(d)\right\} y-x^{*}\left\{C(y d)-C(y) d^{*}\right\} \quad(\text { by }(1.2)) } \\
&=\{\hat{C}(x d)-d \hat{C}(x)\} y+x^{*} C(y) d^{*}+x^{*} C\left(d^{*} y^{*}\right) \quad(\text { by }(2.14),(1.4)) \\
&=\left\{\hat{C}\left(x d y^{*}\right)+C\left(y^{*}\right)^{*} x d\right\}-d \hat{C}(x) y+\left\{x^{*} C(y)\right\} d^{*} \\
&+\left\{\hat{C}\left(x d^{*} y^{*}\right)-d^{*} y^{*} \hat{C}(x)\right\} \quad(\text { by }(2.14)) \\
&= T(d)\left\{\hat{C}\left(x y^{*}\right)-y^{*} \hat{C}(x)-x^{*} C\left(y^{*}\right)\right\}+d\left\{-\hat{C}(x) y+y^{*} \hat{C}(x)\right\} \\
&+\left\{-C(y)^{*} x-x^{*} C(y)\right\} d \quad(\text { by }(1.4)) \\
&= 0+d\{T(y) \hat{C}(x)-\hat{C}(x) \circ y-N(C(y), x)\} \quad(\text { by }(2.14)) \\
&= 0 \quad\left(\text { by }\left(0.10^{\prime}\right),(1.7),(2.10)\right) .
\end{aligned}
$$

3. Cayley derivations of $\mathbf{C}(A, \mu)$. In this section we describe how Cayley derivations of $\mathbf{C}(A, \mu)$ are built out of Cayley derivations and anti-derivations of $A$. In most cases of dimension $\geq 8$ there are no Cayley derivations at all.

3.1. Lemma. $A$ map $D_{+}(a+b l)=D_{11}(a)+D_{22}(b) l$ is a Cayley derivation of $\mathbf{C}(A, \mu)$ iff

(i) $D_{11}=D_{0}$ is a Cayley derivation of $A$

(ii) $D_{22}(a)=d_{0} a^{*}-D_{0}(a)$ 
(iii) $\left[D_{0}(a), b\right]=d_{0}(a b)-\left(d_{0} b\right) a$

(iv) $d_{0} \in \operatorname{Comm}(A)$ has $d_{0}[a, b]=\left[a, d_{0}, b\right]$ and $3 d_{0}[A, A]=0$ for all $a, b \in A$.

Proof. For $x_{i}=a_{i}+b_{i} l$ we have

$$
D\left(x_{1} x_{2}\right)=D_{11}\left(a_{1} a_{2}+\mu b_{2}^{*} b_{1}\right)+D_{22}\left(b_{1} a_{2}^{*}+b_{2} a_{1}\right) l
$$

and

$$
\begin{aligned}
D\left(x_{1}\right) x_{2}^{*} & +D\left(x_{2}\right) x_{1} \\
= & \left\{D_{11}\left(a_{1}\right)+D_{22}\left(b_{1}\right) l\right\}\left\{a_{2}^{*}-b_{2} l\right\} \\
& +\left\{D_{11}\left(a_{2}\right)+D_{22}\left(b_{2}\right) l\right\}\left\{a_{1}+b_{1} l\right\} \\
= & \left\{D_{11}\left(a_{1}\right) a_{2}^{*}+D_{11}\left(a_{2}\right) a_{1}\right\}+\mu\left\{-b_{2}^{*} D_{22}\left(b_{1}\right)+b_{1}^{*} D_{22}\left(b_{2}\right)\right\} \\
& +\left\{-b_{2} D_{11}\left(a_{1}\right)+D_{22}\left(b_{2}\right) a_{1}^{*}\right\} l \\
& +\left\{D_{22}\left(b_{1}\right) a_{2}+b_{1} D_{11}\left(a_{2}\right)\right\} l,
\end{aligned}
$$

so (using cancellability of $\mu$ ) $D_{+}$is a Cayley derivation iff $D_{11}=D_{0}$ is a Cayley derivation of $A$ and for all $a, b \in A$

$$
\begin{aligned}
& D_{0}\left(b_{2}^{*} b_{1}\right)=-b_{2}^{*} D_{22}\left(b_{1}\right)+b_{1}^{*} D_{22}\left(b_{2}\right) \\
& D_{22}\left(b_{2} a_{1}\right)=D_{22}\left(b_{2}\right) a_{1}^{*}-b_{2} D_{0}\left(a_{1}\right) \\
& D_{22}\left(b_{1} a_{2}^{*}\right)=D_{22}\left(b_{1}\right) a_{2}+b_{1} D_{0}\left(a_{2}\right)
\end{aligned}
$$

Here (3) is superfluous in the presence of (2): if we replace $b_{i}$ by $b, a_{i}$ by $a$ then $(2)+(3)$ becomes $D_{22}(b T(a))=D_{22}(b) T(a)$, which holds automatically. Setting $b_{2}=1$ in (2) yields (ii) for $d_{0}=D_{22}(1)$. Setting $b_{1}=1$ in (1) yields $D_{0}\left(b^{*}\right)=-b^{*} d_{0}+d_{0} b^{*}-D_{0}(b)=\left[d_{0}, b^{*}\right]+D_{0}\left(b^{*}\right)$ (by (1.4)), and $\left[d_{0}, A\right]=0$ is the definition (0.6) of $d_{0}$ belonging to $\operatorname{Comm}(A)$. Condition (2) reduces to

$$
\begin{aligned}
0 & =\left\{d_{0} b^{*}-D_{0}(b)\right\} a^{*}-b D_{0}(a)-\left\{d_{0}(b a)^{*}-D_{0}(b a)\right\} \\
& =\left(d_{0} b^{*}\right) a^{*}-d_{0}\left(a^{*} b^{*}\right)+\left[D_{0}(a), b\right] \quad(\text { by }(1.2)) \\
& =\left(d_{0} b^{*}\right) a^{*}-d_{0}\left(a^{*} b^{*}\right)+\left[D_{0}\left(a^{*}\right), b^{*}\right] \quad(\text { by }(1.4)),
\end{aligned}
$$

which is just (iii). Then $(1)+(2)$ reduces to

$$
\begin{aligned}
0= & \left\{D_{0}\left(b^{*} a\right)+b^{*}\left(d_{0} a^{*}-D_{0}(a)\right)-a^{*}\left(d_{0} b^{*}-D_{0}(b)\right)\right\} \\
& +\left\{d_{0}\left(b^{*} a\right)^{*}-D_{0}\left(b^{*} a\right)-\left(d_{0} b-D_{0}\left(b^{*}\right)\right) a^{*}+b^{*} D_{0}(a)\right\} \\
= & b^{*}\left(d_{0} a^{*}\right)-a^{*}\left(d_{0} b^{*}\right)+\left[a^{*}, D_{0}(b)\right]+d_{0}\left(a^{*} b\right) \\
& -\left(d_{0} b\right) a^{*} \quad(\text { by }(1.4))
\end{aligned}
$$




$$
\begin{aligned}
= & T(b)\left[d_{0}, a^{*}\right]-b\left(d_{0} a^{*}\right)-a^{*}\left(d_{0} b\right)+\left\{-d_{0}\left(b a^{*}\right)+\left(d_{0} a^{*}\right) b\right\} \\
& +d_{0}\left(a^{*} b\right)-\left(d_{0} b\right) a^{*} \quad(\text { by }(\mathrm{iii})) \\
= & d_{0}\left[a^{*}, b\right]+\left[d_{0} a^{*}, b\right]+T(b)\left[d_{0}, a^{*}\right]-\left[d_{0} b, a^{*}\right] \\
= & -d_{0}\left[a^{*}, b^{*}\right]-\left[d_{0} a^{*}, b^{*}\right]+\left[d_{0} b^{*}, a^{*}\right],
\end{aligned}
$$

so replacing $a$ by $a^{*}, b$ by $b^{*}$ it becomes

(iv) ${ }^{\prime} d_{0}[a, b]+\left[d_{0} a, b\right]+\left[a, d_{0} b\right]=0$.

Now (iii) can be rewritten (using $\left[d_{0}, A\right]=0$ ) as

(iii) $^{\prime}\left[D_{0}(a), b\right]=d_{0}[a, b]-\left[d_{0}, b, a\right]=\left[a, b, d_{0}\right]-\left[d_{0}, b, a\right]$.

Since $[x, y]^{*}=\left[y^{*}, x^{*}\right]=[y, x]=-[x, y],[x, y, z]^{*}=-\left[z^{*}, y^{*}, x^{*}\right]=$ $[z, y, x]$ for any scalar involution, we see $\left[a, b, d_{0}\right]=\left[D_{0}(a), b\right]+\left[d_{0} b, a\right]$ is skew, so

$$
\begin{aligned}
d_{0}[a, b]-\left[a, d_{0} b\right] & =\left[a, b, d_{0}\right]+\left[d_{0}, b, a\right] \quad\left(\text { by }(\text { iii })^{\prime}\right) \\
& =T\left(\left[a, b, d_{0}\right]\right)=0,
\end{aligned}
$$

hence $d_{0}[a, b]-\left[d_{0} a, b\right]=0$ too by skewness in $a, b$, so

(iva) $d_{0}[a, b]=\left[d_{0} a, b\right]=\left[a, d_{0} b\right]$

and (iv)' becomes

(ivb) $3 d_{0}[a, b]=0$.

Thus (1)-(3) are equivalent to (i)-(iv).

3.2. Lemma. $D_{-}(a+b l)=D_{12}(b)+D_{21}(a) l$ is a Cayley derivation of $\mathbf{C}(A, \mu)$ iff

(i) $D_{21}=C_{0}$ is a Cayley anti-derivation of $A$

(ii) $D_{12}=L_{c_{0}}-\mu C_{0}$

(iii) $\mu\left[C_{0}(a), b\right]=\left[c_{0}, b, a\right]$

(iv) $c_{0} \in \operatorname{Comm}(A)$ has $c_{0}[a, b]=\left[c_{0} a, b\right]+\left[a, c_{0} b\right]$ for all $a, b \in A$.

Proof. $D_{-}$restricts and projects to a Cayley derivation of $A$ into $A l$; since $a l \rightarrow a$ is an isomorphism $A l \rightarrow A^{\text {op }}$ of right $A$-modules, we see $D_{21}$ : $A \rightarrow A^{\mathrm{op}}$ is a Cayley derivation, i.e. $D_{21}$ is a Cayley anti-derivation $C_{0}$ of $A$. Then $D_{-}\left(x_{1} x_{2}\right)=D_{12}\left(b_{1} a_{2}^{*}+b_{2} a_{1}\right)+C_{0}\left(a_{1} a_{2}+\mu b_{2}^{*} b_{1}\right) l$ and

$$
\begin{aligned}
D_{-} & \left(x_{1}\right) x_{2}^{*}+D_{-}\left(x_{2}\right) x_{1} \\
= & \left\{D_{12}\left(b_{1}\right)+C_{0}\left(a_{1}\right) l\right\}\left\{a_{2}^{*}-b_{l}\right\}+\left\{D_{12}\left(b_{2}\right)+C_{0}\left(a_{2}\right) l\right\}\left\{a_{1}+b_{1} l\right\} \\
= & \left\{D_{12}\left(b_{1}\right) a_{2}^{*}+\mu b_{1}^{*} C_{0}\left(a_{2}\right)\right\}+\left\{-\mu b_{2}^{*} C_{0}\left(a_{1}\right)+D_{12}\left(b_{2}\right) a_{1}\right\} \\
& +\left\{-b_{2} D_{12}\left(b_{1}\right)+b_{1} D_{12}\left(b_{2}\right)\right\} l+\left\{C_{0}\left(a_{1}\right) a_{2}+C_{0}\left(a_{2}\right) a_{1}^{*}\right\} l,
\end{aligned}
$$

so $D_{-}$is a Cayley derivation iff

$$
\mu C_{0}\left(b_{2}^{*} b_{1}\right)=-b_{2} D_{12}\left(b_{1}\right)+b_{1} D_{12}\left(b_{2}\right)
$$




$$
D_{12}\left(b_{1} a_{2}^{*}\right)=D_{12}\left(b_{1}\right) a_{2}^{*}+\mu b_{1}^{*} C_{0}\left(a_{2}\right)
$$

$$
D_{12}\left(b_{2} a_{1}\right)=-\mu b_{2}^{*} C_{0}\left(a_{1}\right)+D_{12}\left(b_{2}\right) a_{1} .
$$

Here (2) and (3) are equivalent since (2) $+(3)$ is $D_{12}(b T(a))=D_{12}(b) T(a)$, which holds automatically. If we set $c_{0}=D_{12}(1)$ then (3) implies $D_{12}(a)$ $=c_{0}(a)-\mu C_{0}(a)$, i.e. $D_{12}=L_{c_{0}}-\mu C_{0}$ as in (ii). Thus (3) reduces to

$$
\begin{aligned}
0 & =c_{0}(b a)-\mu C_{0}(b a)+\mu b^{*} C_{0}(a)-\left(c_{0} b\right) a+\mu C_{0}(b) a \\
& =-\left[c_{0}, b, a\right]-\mu\left[C_{0}(a), b^{*}\right]=\mu\left[C_{0}(a), b\right]-\left[c_{0}, b, a\right]
\end{aligned}
$$

as in (iii). Setting $b_{2}=1$ in (1) shows $c_{0} b_{1}=b_{1} c_{0}$, i.e. $c_{0} \in \operatorname{Comm}(A)$, so $(1)+(3)$ reduces to

$$
\begin{aligned}
0= & \left\{\mu C_{0}(b a)+b^{*}\left(c_{0} a\right)-\mu b^{*} C_{0}(a)-a\left(c_{0} b^{*}\right)+\mu a C_{0}\left(b^{*}\right)\right\} \\
& +\left\{c_{0}(b a)-\mu C_{0}(b a)+\mu b^{*} C_{0}(a)-\left(c_{0} b\right) a+\mu C_{0}(b) a\right\} \\
= & \mu\left[C_{0}(b), a\right]-b\left(c_{0} a\right)+a\left(c_{0} b\right)-\left[c_{0}, b, a\right] \\
& +T(b)\left(c_{0} a-a c_{0}\right) \quad(\text { using }(1.4)) \\
= & {\left[c_{0}, a, b\right]-b\left(a c_{0}\right)+\left[a, c_{0} b\right]+(b a) c_{0}+0 \quad \text { (using (iii)) } } \\
= & {\left[c_{0} a, b\right]+\left[a, c_{0} b\right]-c_{0}[a, b] }
\end{aligned}
$$

as in (iv).

Putting these two pieces together, we get

3.3. Cayley Derivation Theorem. When $A$ is an algebra with scalar involution and $\mu$ a cancellable scalar, the Cayley derivations of $\mathrm{C}(A, \mu)$ are precisely all

$$
D=\left(\begin{array}{ll}
D_{0} & L_{c_{0}}-C_{0} \\
C_{0} & L_{d_{0}} J-D_{0}
\end{array}\right)
$$

where $J(x)=x^{*}$ and for all $a, b \in A$

(i) $D_{0}$ is a Cayley derivation of $A$

(ii) $\left[D_{0}(a), b\right]=d_{0}(a b)-\left(d_{0} b\right) a$

(3.5) (iii) $d_{0} \in \operatorname{Comm}(A)$ has $d_{0}[a, b]=\left[a, d_{0} b\right]$ and $3 d_{0}[A, A]=0$

(iv) $C_{0}$ is a Cayley anti-derivation of $A$

(v) $\mu\left[C_{0}(a), b\right]=\left[c_{0}, b, a\right]$

(vi) $c_{0} \in \operatorname{Comm}(A)$ has $c_{0}[a, b]=\left[c_{0} a, b\right]+\left[a, c_{0} b\right]$. 
Proof. As in (2.3), and

$$
D=\left(\begin{array}{ll}
D_{11} & D_{12} \\
D_{21} & D_{22}
\end{array}\right)
$$

is graded, where

$$
D_{+}=\left(\begin{array}{ll}
D_{11} & 0 \\
0 & D_{22}
\end{array}\right)
$$

is a derivation iff $D_{11}=D_{0}, D_{22}=L_{d_{0}} J-D_{0}$ as in (i)-(iii) by Lemma 3.1, and

$$
D_{-}=\left(\begin{array}{ll}
0 & D_{12} \\
D_{21} & 0
\end{array}\right)
$$

is a derivation iff $D_{21}=C_{0}, D_{12}=L_{c_{0}}-\mu C_{0}$ as in (iv)-(vi) by Lemma 3.2 .

3.4. Corollary. If $A$ is unitally rigid and

(i) $\operatorname{Comm}(A)=\Phi 1$

(ii) $\lambda A \subset \Phi 1 \Rightarrow \lambda=0$ (e.g. if $A$ has cancellable commutators)

then when $\Phi$ has no 3-torsion there are no Cayley derivations

$$
\text { Cayder }(\mathbf{C}(A, \mu))=0
$$

while if $3 \Phi=0$ then

Cayder $(\mathbf{C}(A, \mu))=\Phi S \quad(S$ standard skew Cayley map $)$.

Proof. If $\operatorname{Comm}(A)=\Phi 1$ then in (3.3) $d_{0}=\delta 1, c_{0}=\gamma 1 \in \Phi 1$ and (ii) becomes $\left[D_{0}(a), b\right]=\delta[a, b], \quad D_{0}(a)-\delta a \in \operatorname{Comm}(A)=\Phi 1$, so $\left(D_{0}-\delta\right)(A) \subset \Phi 1$; similarly (3.3)(iii) becomes $3 \delta[A, A]=0$ (i.e. $3 \delta A \subset$ $\Phi 1)$, (v) becomes $\left[C_{0}(a), b\right]=0$ (cancelling $\mu$ ) so $C_{0}(a) \in \operatorname{Comm}(A)=$ $\Phi 1$ and $C_{0}(A) \subset \Phi 1$, while (vi) becomes $\gamma[a, b]=2 \gamma[a, b]$, so $\gamma[A, A]=0$ (i.e. $\gamma A \subset \Phi 1$ ). If $A$ is unitally rigid we have $C_{0}=0$ by (1.9)(ii). If 3.4(ii) holds we see $\gamma=3 \delta=0$, so if $\Phi$ has no 3-torsion then $\delta=0$ too; then $D_{0}(A) \subset \Phi 1$ forces $D_{0}=0$ by (1.9)(ii), therefore when $A$ has no 3-torsion $D=0$. When $3 \Phi=0$ we know by (1.8), (1.10)(ii) that $D_{0}=\delta S_{0}$,

$$
\begin{aligned}
D(x) & =D(a+b l)=\delta S_{0}(a)+\delta\left(b^{*}-S_{0}(b)\right) l \\
& =\delta\left\{a^{*}-a+\left(b^{*}-\left(b^{*}-b\right)\right) l\right\} \\
& =\delta\left\{a^{*}-a-2 b l\right\} \quad(\text { since } 3 b=0) \\
& =\delta\left\{\left(a^{*}-b l\right)-(a+b l)\right\}=\delta\left\{x^{*}-x\right\}=\delta S(x) .
\end{aligned}
$$


4. Derivations of generalized Cayley-Dickson algebras. Our calculations simplify greatly in the case of generalized Cayley-Dickson algebras. These algebras are always unitally rigid, and by $(0.7)$ have cancellable commutators and $\operatorname{Comm}(A)=\Phi 1$ and $N(x, y)$ nondegenerate in dimension $\geq 4$, have $N(A)=\Phi 1$ in dimension $\geq 8$, and all derivations are traceless by (0.13). The description of derivations given in 2.4 simplifies to

4.1. Schafer Derivation Theorem [5]. Let $\mathbf{C}_{n}=\mathbf{C}^{n-3}(C)$ be a generalized Cayley-Dickson algebra of dimension $2^{n}(n \geq 3)$ over $\Phi$ obtained from a Cayley algebra $C$. Then if $\Phi$ has no 2-or 3-torsion we have

$$
\text { Cayder }\left(\mathbf{C}_{n}\right)=0, \quad \operatorname{Der}\left(\mathbf{C}_{n}\right)=\widetilde{\operatorname{Der}(C)}
$$

while if $2 \Phi=0$ then

$$
\begin{aligned}
\text { Cayder }\left(\mathbf{C}_{n}\right) & =0, \\
\operatorname{Der}\left(\mathbf{C}_{n}\right) & =\widetilde{\operatorname{Der}(C)} \boxplus \Phi Z_{4} \boxplus \cdots \boxplus \Phi Z_{n} \text { for central } Z_{i}
\end{aligned}
$$

and if $3 \Phi=0$ then

$$
\text { Cayder }\left(\mathbf{C}_{n}\right)=\Phi S_{n},
$$

$$
\operatorname{Der}\left(\mathbf{C}_{n}\right)=\widetilde{\operatorname{Der}(C)} \boxplus \Phi W_{4} \boxplus \cdots \boxplus \Phi W_{n} \text { for central } W_{i}
$$

Proof. Let $\mathbf{C}_{n}=\mathbf{C}(A, \mu)$ for $A=\mathbf{C}_{n-1}$ generalized Cayley-Dickson of dimenson $2^{n-1} \geq 4$. By Corollary 3.4 we have Cayley $\left(\mathbf{C}_{n}\right)=0$ if $\Phi$ has no 3-torsion, and Cayder $\left(\mathbf{C}_{n}\right)=\Phi S_{n}\left(S_{n}(x)=x^{*}-x\right)$ if $3 \Phi=0$. The derivation statement $\operatorname{Der}\left(\mathbf{C}_{n}\right)=\widehat{\operatorname{Der}(C)}$ is trivial if $n=3\left(\mathbf{C}_{n}=C\right)$, so assume $n \geq 4, \operatorname{dim} A \geq 8$. Then $N(A)=\Phi 1, N(x, y)$ is nondegenerate; if $\Phi$ has no 3-torsion then $\operatorname{Cayder}(A)=0$ by the above, so we can apply Corollary 2.8 to see $\operatorname{Der}\left(\mathbf{C}_{n}\right)=\widehat{\operatorname{Der}_{0}(A)}=\widehat{\operatorname{Der}(A)}$ (hence $\operatorname{Der}\left(\mathbf{C}_{n}\right)$ $=\widehat{\operatorname{Der}(C)}$ as in (i) by induction) if $\Phi$ also has no 2-torsion, whereas if $2 \Phi=0$ then $\operatorname{Der}\left(\mathbf{C}_{n}\right)=\widehat{\operatorname{Der}(A)} \boxplus \Phi Z_{n}$ (hence $\operatorname{Der}\left(\mathbf{C}_{n}\right)=\widehat{\operatorname{Der}(C)} \boxplus$ $\Phi Z_{4} \boxplus \cdots \boxplus \Phi Z_{n}$ as in (ii) by induction) for central $Z_{n}(a+b l)=b l$. If $3 \Phi=0$ we have Cayder $(A)=\Phi S$ by the above, so Corollary 2.8 says $\operatorname{Der}\left(\mathbf{C}_{n}\right)=\widetilde{\operatorname{Der}(A)} \boxplus \Phi W_{n}$ (hence $\operatorname{Der}\left(\mathbf{C}_{n}\right)=\widetilde{\operatorname{Der}(C)} \boxplus \Phi W_{4} \boxplus \cdots \boxplus$ $\Phi W_{n}$ as in (iii) by induction) for central $W_{n}(a+b l)=\mu S(b)+S(a) l$.

The natural matrix form for the $Z_{i}$ in (ii) is a string of $2^{n+1-l}$ blocks down the diagonal, alternating between the $2^{t-1} \times 2^{t-1}$ zero block and the $2^{i-1} \times 2^{i-1}$ identity block. The natural matrix for the $W_{i}$ in (iii) is a string 
of $2^{n+1-i}$ blocks down the superdiagonal and subdiagonal; on the subdiagonal the blocks are alternatingly the $2^{i-1} \times 2^{i-1}$ matrix of $S=I+J$ and the $2^{i-1} \times 2^{i-1}$ zero block, while the superdiagonal is just $\mu_{i}$ times the subdiagonal $\left(\mathbf{C}_{i-3}=\mathbf{C}\left(\mathbf{C}_{i-4}, \mu_{i}\right)\right)$.

Over a field of characteristic $\neq 3, \operatorname{Der}(C)$ is a simple Lie algebra of type $G_{2}$.

\section{REFERENCES}

[1] A. A. Albert, Quadratic forms permitting composition, Annals. of Math., 43 (1942), 161-177.

[2] R. B. Brown, On generalized Cayley-Dickson algebras, Pacific J. Math., 20 (1967), 415-422.

[3] R. Erdmann, Über verallgemeinerte Cayley-Dickson Algebren, J. für Reine und Angew Math., 250 (197), 153-181.

[4] K. McCrimmon, Nonassociative algebras with scalar involution, to appear.

[5] R. D. Schafer, On the algebras formed by the Cayley-Dickson process, Amer. J. Math., 76 (1954), 435-446. [6] $\frac{}{64-69}$. On the simplicity of the Lie algebra $E_{7}$ and $E_{8}$, Indag. Math., 28 (1966),

Received April 18, 1983. The author wishes to thank the NSF and NRC for financial support during the preparation of this paper, and the University of British Columbia for its hospitality.

UNIVERSITY OF VIRGINIA

Charlottesville, VA 22903 


\section{PACIFIC JOURNAL OF MATHEMATICS EDITORS}

DONALD BABBITT (Managing Editor)
University of California
Los Angeles, CA 90024
CHARLES R. DePrima
California Institute of Technology
Pasadena, CA 91125
R. FinN
Stanford University
Stanford, CA 94305

HERMANN FLASCHKA

University of Arizona

Tucson, AZ 85721

RAMESH A. GANGOLLI

University of Washington

Seattle, WA 98195

ROBION KIRBY

University of California

Berkeley, CA 94720

C. C. MOore

University of California

Berkeley, CA 94720
Hugo RossI

University of Utah

Salt Lake City, UT 84112

H. SAMELSON

Stanford University

Stanford, CA 94305

HaRold STARK

University of California, San Diego

La Jolla, CA 92093

\section{ASSOCIATE EDITORS}
R. ARENS
E. F. BECKENBACH (1906-1982)
B. H. NEUMANN
F. WOLF
K. YoshidA

\section{SUPPORTING INSTITUTIONS}

UNIVERSITY OF ARIZONA

UNIVERSITY OF BRITISH COLUMBIA

CALIFORNIA INSTITUTE OF TECHNOLOGY

UNIVERSITY OF CALIFORNIA

MONTANA STATE UNIVERSITY

UNIVERSITY OF NEVADA, RENO

NEW MEXICO STATE UNIVERSITY

OREGON STATE UNIVERSITY
UNIVERSITY OF OREGON

UNIVERSITY OF SOUTHERN CALIFORNIA

STANFORD UNIVERSITY

UNIVERSITY OF HAWAII

UNIVERSITY OF TOKYO

UNIVERSITY OF UTAH

WASHINGTON STATE UNIVERSITY

UNIVERSITY OF WASHINGTON 


\section{Pacific Journal of Mathematics}

\section{Vol. 117, No. $1 \quad$ January, 1985}

Amos Altshuler and Leon Steinberg, The complete enumeration of the

4-polytopes and 3 -spheres with eight vertices $\ldots \ldots \ldots \ldots \ldots \ldots \ldots \ldots$

Michael James Beeson, The $6 \pi$ theorem about minimal surfaces . . . . . . . . 17

Jeffrey Lawrence Caruso and Stefan Waner, An approximation theorem

for equivariant loop spaces in the compact Lie case ...............27

Jo-Ann Deborah Cohen, Topologies on the quotient field of a Dedekind

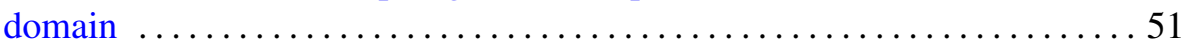

Szymon Dolecki, Gabriele H. Greco and Alois Andreas Lechicki,

Compactoid and compact filters .........................6 69

Roger William Hansell (Sr.), Generalized quotient maps that are

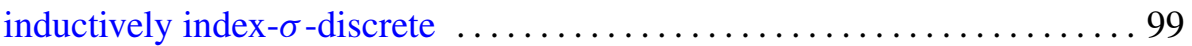

Gerhard Huisken, Capillary surfaces over obstacles $\ldots \ldots \ldots \ldots \ldots \ldots \ldots 121$

Jun Shung Hwang, A problem on continuous and periodic functions . . . . 143

Ronald Fred Levy and Michael David Rice, The extension of equi-uniformly continuous families of mappings $\ldots \ldots \ldots \ldots \ldots \ldots 149$

Kevin Mor McCrimmon, Derivations and Cayley derivations of generalized

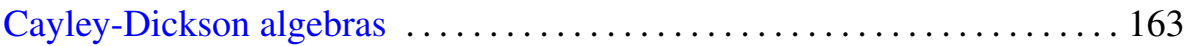

H. M. (Hari Mohan) Srivastava, A multilinear generating function for the Konhauser sets of biorthogonal polynomials suggested by the Laguerre

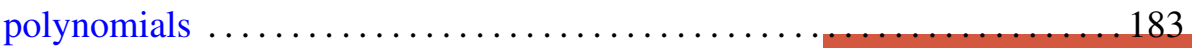

Zhu Jia Lu, Some maximum properties for a family of singular hyperbolic operators 